\title{
然
}

Subdirección General de Estudios y Evaluación de Instrumentos de Politica Comercial*

\section{COMERCIO MINORISTA: RECUPERACIÓN, TRANSFORMACIÓN Y RESILIENCIA}

El comercio minorista es un sector clave, no solo por su contribución a la actividad económica, sino también por su aportación a la cohesión social y territorial. Es una actividad que afronta el reto de la digitalización y que, en el año 2020, se ha visto especialmente afectada por la pandemia COVID-19 y por las medidas de restricción de la movilidad que ha sido necesario adoptar para su contención. En este trabajo se analizan el impacto de la pandemia en el sector, la utilización de las medidas de apoyo que se han puesto en marcha para paliar sus efectos y el diseño de las medidas de acompañamiento que serán precisas para que, a la salida de la crisis, el sector aborde con éxito el desafío de la transformación digital, mantenga su papel de vector de cohesión social y territorial y contribuya a un crecimiento inclusivo y sostenible.

Palabras clave: digitalización, COVID-19, comercio minorista, pagos con tarjeta, restricciones a la movilidad, confinamiento, consumo, políticas públicas.

Clasificación JEL: L81, L88.

\section{Introducción}

El comercio minorista desempeña un papel clave en la actividad económica, ya que conecta a los productores de bienes con los consumidores finales. Facilita que los consumidores dispongan de los bienes que demandan, haciendo posible la satisfacción de sus necesidades, y que los productores vendan su producto y obtengan información sobre la evolución de las necesidades y preferencias de los clientes. Este papel clave se puso

\footnotetext{
* Secretaría de Estado de Comercio.

Versión de febrero de 2021.

DOI: https:/doi.org/10.32796/bice.2021.3132.7161
}

especialmente de manifiesto en el trascurso del año 2020, en el que se ha constatado cómo el comercio minorista posibilitó la provisión de los bienes más básicos durante los momentos más duros de la pandemia, a pesar de las difíciles circunstancias.

La relevancia social del comercio minorista, sin embargo, es más profunda de lo que muestra su papel de canal de comunicación entre consumidor y productor. Las características estructurales del sector, como el predominio de las pymes, su elevada intensidad en trabajo, su contribución al empleo y emprendimiento femeninos, y su distribución territorial determinan que el comercio minorista no solo contribuya a la creación de riqueza y empleo, sino $\triangleright$ 
que sea un vector decisivo de cohesión social $y$ territorial.

En este artículo se revisan algunas de las características del sector en España, la repercusión de la crisis sanitaria asociada a la pandemia COVID-19 en su evolución y los desafíos que deberá afrontar en el futuro inmediato, así como algunas de las medidas de apoyo que ha recibido y las que serán precisas para superar estos retos.

En el epígrafe 2 se presentan los principales rasgos estructurales y en el tercero se sintetizan los retos que afronta el sector. En el epígrafe 4 se analiza el impacto de la COVID-19 en el comercio minorista, mientras que en el quinto se estudia el uso por el sector de las principales medidas de apoyo que se han establecido para atenuar el impacto de la crisis en la actividad económica y salvaguardar el tejido empresarial y el empleo. El artículo finaliza con un apartado en el que se resumen las principales conclusiones, en particular con relación a las actuaciones que deberán ponerse en marcha para favorecer la recuperación del sector y la superación con éxito de los desafíos que afronta.

\section{Estructura del sector}

El comercio minorista es un sector intensivo en empleo, con fuerte predominio de la pyme, que ofrece oportunidades de empleo y emprendimiento de las mujeres, y está presente en todo el territorio nacional. Como se ha señalado, estas características dotan al sector de una particular relevancia, no solo económica, sino también social y territorial.

Así, la contribución del comercio minorista a la generación de empleo se sitúa por encima de su participación en el PIB. La diferencia entre estos porcentajes es indicativa de la intensidad en empleo del sector y de su relevancia social como generador de puestos de trabajo. Según los últimos datos disponibles, el comercio al por menor, excepto de vehículos de motor y motocicletas, división 47 de la Clasificación Nacional de Actividades Económicas (CNAE), generó, en 2018, un valor añadido bruto que representó el 5,2\% del total de la economía. Su contribución al empleo es aún más sustantiva. Con datos de 2019, ocupó a 1.931 .600 personas, lo que supuso el $9,8 \%$ del total de ocupados. El $60,9 \%$ de los ocupados del sector son mujeres y representan un $13,0 \%$ del empleo femenino.

Además, es un sector empresarialmente atomizado. Agrupa a 435.836 empresas, con datos referidos a 1 de enero de 2020, lo que supone el $12,8 \%$ del total de empresas españolas. Este porcentaje, superior a la contribución del sector tanto al PIB como al empleo, revela el predominio de empresas de dimensión relativa reducida.

La comparación de las empresas de comercio minorista por intervalo de tamaño, según su número de asalariados, con las del conjunto de la economía confirma esta percepción. El 98,4\% de las empresas del sector tiene menos de diez asalariados y representan un $13,2 \%$ de las empresas del conjunto de la economía dentro de este estrato. La presencia de empresas de comercio minorista en los estratos de tamaño superiores es sensiblemente más limitada, con tasas de participación sobre el total de las empresas de cada estrato entre el $3,3 \%$ y el $4,7 \%$.

El comercio minorista es, además, un sector generador de oportunidades para el emprendimiento de las mujeres. Más de 304.000 de las empresas del sector (en torno al $70 \%$ del total) tienen como soporte legal a una persona física. De estas empresas, casi 155.000 (el 51,0\%, $\triangleright$ 
CUADRO 1

RASGOS ESTRUCTURALES DEL COMERCIO MINORISTA

\begin{tabular}{|c|c|c|c|}
\hline & Valor & $\begin{array}{l}\text { Porcentaje sobre el total } \\
\text { de la economía }\end{array}$ & Año de referencia \\
\hline Valor añadido bruto (millones de euros). & 56.509 & 5,2 & 2018 \\
\hline Ocupados (personas). & 1.931 .600 & 9,8 & 2019 \\
\hline Mujeres & 1.176 .500 & 13,0 & 2019 \\
\hline Hombres & 755.100 & 7,0 & 2019 \\
\hline Total empresas (unidades) ...... & 435.836 & 12,8 & 2020 \\
\hline Menos de 10 asalariados & 428.869 & 13,2 & 2020 \\
\hline De 10 a 49 asalariados & 6.028 & 4,7 & 2020 \\
\hline De 50 a 249 asalariados & 713 & 3,3 & 2020 \\
\hline Más de 250 asalariados ... & 226 & 4,7 & 2020 \\
\hline Empresas con soporte legal persona física (unidades) ....................... & 304.083 & 16,0 & 2020 \\
\hline Mujeres & 154.971 & 22,2 & 2020 \\
\hline Hombres & 149.112 & 12,4 & 2020 \\
\hline Establecimientos (unidades) .... & 550.310 & 14,1 & 2020 \\
\hline
\end{tabular}

frente al 36,7\% en el conjunto de la economía) están lideradas por mujeres. Así, el comercio minorista aporta un $22 \%$ al total de empresas de la economía española que tienen como soporte legal a una persona física y son de titularidad femenina. Este porcentaje es notablemente superior al $12,4 \%$ correspondiente a los hombres.

El papel de cohesión social y territorial del comercio minorista se pone de manifiesto también en su extensa red de establecimientos, que dota al sector de la necesaria capilaridad para poner en contacto oferta y demanda. Cada empresa del sector tiene, en promedio, 1,3 establecimientos, lo que da como resultado un total de 550.310 establecimientos de comercio minorista, el $14,1 \%$ del total de los que existen en España.

\section{El reto de la digitalización}

El comercio al por menor se enfrenta en la actualidad a un desafío relevante: la transformación digital. El despegue del comercio electrónico es, quizá, la característica más visible de este desafío. Los rasgos estructurales del sector antes descritos deben tenerse presentes para valorar la capacidad de las empresas para acometer este reto, sus implicaciones económicas y sociales, y el diseño de las medidas de apoyo precisas para acompañarlas.

El fuerte crecimiento del comercio electrónico en la última década refleja la creciente penetración de esta modalidad de compra tanto entre los consumidores como entre las empresas españolas. Este hecho revela un profundo cambio estructural al que debe adaptarse el sector de comercio minorista. Así, según datos del Observatorio de las Telecomunicaciones y de la Sociedad de la Información (ONTSI), el volumen de comercio electrónico entre empresas y consumidores (B2C) superó los 50.000 millones de euros en 2019. En la última década, esta cifra se ha multiplicado por 6,5 y ha registrado, todos los años, tasas de crecimiento de dos dígitos (Gráfico 1).

El número de personas que realizan compras electrónicas y el importe que destinan a este canal de compra están también $\triangleright$ 


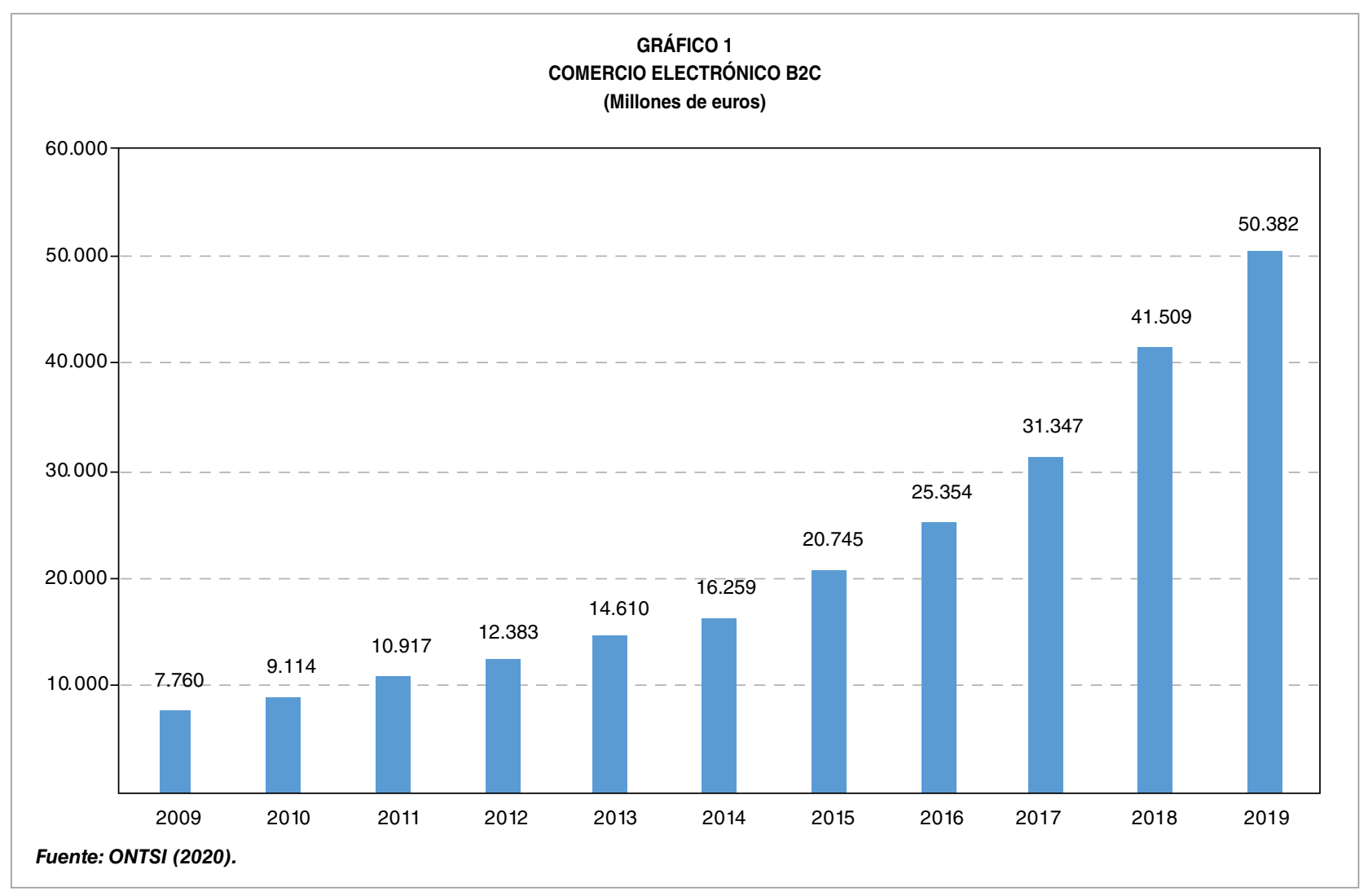

registrando un fuerte aumento, y se adquiere, por esta vía, una variedad de productos cada vez más amplia. Así, en 2019, 24,3 millones de personas compraron online en España, con un promedio de 29,5 compras online anuales a las que destinaron un total de unos 2.076 euros por persona, más del doble que cuatro años antes. Las categorías de productos más demandados, en términos de número de compradores, incluyeron ropa, calzado y complementos (el $59,2 \%$ de los compradores adquirieron, entre otros, estos productos); reservas de alojamiento y paquetes turísticos $(52,1 \%)$, entradas a espectáculos y eventos (49,0\%), billetes de transporte $(45,3 \%)$ y comida a domicilio $(38,5 \%)$. En términos de gasto, el turismo y el transporte fueron las categorías que lideraron este canal, con participaciones del 18,9\% $y$ del $12,0 \%$ en el gasto total, respectivamente (ONTSI, 2020). Es probable que las participaciones de los productos vinculados al turismo, el transporte y los espectáculos y eventos se hayan reducido significativamente durante 2020 , como resultado del especial impacto de la crisis sanitaria en estas actividades.

Desde la perspectiva de la oferta, también son cada vez más las empresas que utilizan el canal electrónico para hacer llegar sus productos o servicios a sus clientes (Cuadro 2). Se observa, además, que las empresas de mayor tamaño utilizan en mayor proporción las ventas electrónicas y que este uso es también más acusado entre las empresas comerciales. Así, en el año 2019, el 25,5\% de las empresas con diez o más empleados $(20,4 \%$ en 2018$)$ y el $9,5 \%$ de las de menos de diez empleados $(5,8 \%$ en 2018) realizaron ventas mediante comercio electrónico. En los sectores comerciales (incluyendo tanto el comercio al por menor como el mayorista y las ventas de vehículos, ya que $\square$ 
CUADRO 2

COMERCIO ELECTRÓNICO Y USO DE LAS TIC EN LAS EMPRESAS 2019-2020

(Porcentajes de empresas)

\begin{tabular}{|c|c|c|c|c|c|c|c|c|}
\hline \multirow[b]{2}{*}{ Tamaño (empleados) } & \multicolumn{2}{|c|}{ Ventas electrónicas (1) } & \multicolumn{2}{|c|}{ Internet y web (2) } & \multicolumn{2}{|c|}{ Facturación (3) } & \multicolumn{2}{|c|}{ Big Data (4) } \\
\hline & Total & Comercio (5) & Total & Comercio (5) & Total & Comercio (5) & Total & Comercio (5) \\
\hline Menos de 10 & 9,5 & nd & 28,8 & nd & 9,2 & nd & 3,1 & nd \\
\hline 10 o más ... & 25,5 & 36,1 & 78,1 & 79,8 & 35,1 & 33,4 & 8,5 & 8,6 \\
\hline De 10 a 49 & 23,3 & 33,2 & 75,6 & 78,1 & 32,0 & 30,6 & 6,8 & 6,9 \\
\hline De 50 a 249 & 33,8 & 49,6 & 88,7 & 88,3 & 47,2 & 47,1 & 13,8 & 16,4 \\
\hline De 250 y más & 45,4 & 70,3 & 95,3 & 95,5 & 65,4 & 59,5 & 28,5 & 30,5 \\
\hline \multicolumn{9}{|c|}{ (1) Realizaron ventas electrónicas (2019). } \\
\hline \multicolumn{9}{|c|}{ (2) Disponían de conexión a internet y sitio/página web (primer trimestre de 2020). } \\
\hline \multicolumn{9}{|c|}{ (3) Enviaron facturas electrónicas que permiten su procesamiento automático (primer trimestre 2020). } \\
\hline \multicolumn{9}{|c|}{ (4) Analizaron grandes fuentes de datos para su empresa, con sus empleados o a través de una empresa externa (primer trimestre 2020). } \\
\hline \multicolumn{9}{|c|}{ (5) CNAE 45, 46, y 47. Incluye venta y reparación de vehículos de motor, comercio al por mayor y comercio al por menor. } \\
\hline \multicolumn{9}{|c|}{ Fuente: INE, Encuesta de uso de TIC y del comercio electrónico en las empresas 2019 y primer trimestre de 2020.} \\
\hline
\end{tabular}

esta fuente no ofrece información desagregada para el comercio minorista) este porcentaje es incluso más elevado, alcanzando el $36,1 \%$ para las empresas de diez o más empleados. No se dispone de datos desagregados sectorialmente para las empresas de menos de 10 empleados, donde se sitúa un elevado porcentaje de las empresas de comercio al por menor.

Estos datos son un indicador de cómo el comercio minorista, al igual que el resto de la economía, está afrontando el reto de la digitalización, del que el auge del comercio electrónico es solo una manifestación parcial. La digitalización de las empresas se asocia con el uso de tecnologías digitales (las que permiten registrar informaciones o datos en forma de números) para mejorar las distintas funciones que se realizan en la empresa. Abarca ámbitos tan diversos como las operaciones comerciales, el marketing, la gestión de clientes o proveedores, la comunicación interna o externa, o los propios procesos de fabricación.

La digitalización del comercio minorista es, a su vez, la vía por la que avanza la progresiva capitalización y automatización del sector. Este proceso es consecuencia de la combinación de dos causas: el cambio tecnológico y el crecimiento de los costes relativos del trabajo frente a los del capital. El proceso de capitalización del sector ya se encuentra avanzado en España desde hace décadas, aunque la evidencia sugiere que existe aún un margen de automatización por recorrer frente a países de nuestro entorno. A título ilustrativo, en España, las estaciones de servicio automáticas suponían en 2020 en torno al 10\% del total, según la Asociación Nacional de Estaciones de Servicio Automáticas (Europapress, 2020), frente al $75 \%$ que representaban en Dinamarca o al $67 \%$ en Suecia, con datos de 2018 (Németh, 2020).

El análisis completo del grado de digitalización de las empresas del sector de comercio minorista excede el alcance de este trabajo, por lo que solo se van a ofrecer algunos datos ilustrativos de los avances registrados y el camino por recorrer. El lector que lo desee puede profundizar en esta cuestión en la Subdirección General de Comercio Internacional de Servicios y Comercio Digital (2019). La evidencia disponible muestra, en todo caso, que la incorporación de tecnologías digitales es una realidad creciente en la economía española y que son las empresas de mayor tamaño las $\triangle$ 
que en mayor medida están incorporando dichas tecnologías.

La Encuesta de uso de tecnologías de la información y la comunicación (TIC) y del comercio electrónico en las empresas, que realiza el Instituto Nacional de Estadística (INE), ofrece una amplia variedad de indicadores que así lo ponen de manifiesto, desde el uso de ordenadores, la contratación de especialistas en TIC o la conexión a internet de las empresas hasta el uso de la impresión 3D o de técnicas de análisis de big data. En el Cuadro 2 se recoge, a título ilustrativo, una selección de indicadores que incluye algunas de las prácticas más generalizadas, como la conexión a internet y el uso de páginas web, y otras menos extendidas, como la facturación electrónica o el uso de análisis de big data, técnica de introducción relativamente reciente.

Estos indicadores revelan que las empresas del sector comercio de mayor tamaño (con diez o más empleados) utilizan las tecnologías TIC, al menos las de aplicación más generalizada, en porcentajes similares a los del conjunto de la economía. Se observa también que el porcentaje de uso crece con el tamaño de la empresa, tanto para el sector comercio como para el conjunto de la economía, y que las microempresas del conjunto de la economía, definidas como las que tienen menos de diez empleados, se sitúan a gran distancia de las empresas de mayor tamaño. Del sector comercial no se dispone de datos para este intervalo de tamaño, pero puede intuirse un comportamiento similar. De nuevo, es preciso recordar que el $98,4 \%$ de las empresas de comercio minorista tiene menos de diez asalariados.

En definitiva, ya antes de 2020, el sector de comercio minorista estaba afrontando dos grandes retos, la transformación digital y, como parte de ella, el auge del comercio electrónico. La crisis sanitaria, cuyo impacto en el sector se analiza en el siguiente epígrafe, ha acentuado la urgencia por acometer las transformaciones necesarias, dado que la percepción del riesgo sanitario, las limitaciones a la movilidad para reducir este riesgo y la propia adaptación del tejido empresarial a la nueva realidad han acelerado las tendencias de cambio que ya estaban presentes.

Las características estructurales del sector de comercio minorista y, en particular, el fuerte predominio de las pymes implican que las empresas de este sector pueden tener que hacer frente a mayores dificultades que las de otros ámbitos para afrontar este desafío.

\section{El impacto de la COVID-19}

El sector de comercio minorista ha acusado el impacto de la pandemia COVID-19. Este efecto, que se ha percibido tanto en los indicadores de actividad del sector como en los de empleo, ha sido geográficamente diferenciado en función de factores relacionados con la evolución de la pandemia, las medidas de contención adoptadas o la dependencia territorial de la actividad turística. Aunque los descensos han sido generalizados por tipo de establecimientos comerciales, existen diferencias significativas entre unos y otros formatos que intensifican tendencias que se percibían en el sector con anterioridad a la crisis sanitaria.

En este epígrafe se presentan indicadores de coyuntura procedentes de diversas fuentes que reflejan el impacto de la crisis sanitaria en el comercio minorista. El análisis se centra en el impacto en la actividad y el empleo, atendiendo también a la dispersión geográfica de dicho impacto. Se completa con una selección de indicadores de alta frecuencia (datos diarios) sobre evolución del número de $\triangleright$ 
contagios, movilidad y gasto mediante tarjetas de crédito que ilustran la relación entre la situación sanitaria, las restricciones a la movilidad y sus efectos en el comercio minorista. El lector interesado en la evolución de los indicadores de comercio interior puede consultar el Cuaderno de Indicadores de Comercio Interior (Subdirección General de Estudios y Evaluación de Instrumentos de Política Comercial, 2021) que se actualiza y publica con periodicidad mensual.

El Gráfico 2 muestra las tasas de variación anuales de las ventas del comercio minorista a precios constantes, de acuerdo con el índice de comercio al por menor. Estas ventas, con la serie corregida de efectos estacionales y de calendario, registraron un descenso del $7,1 \%$ en el conjunto de 2020 que contrasta con el crecimiento del $2,3 \%$ en el ejercicio precedente. Interesa destacar que el impacto de la COVID-19 ha sido heterogéneo en los distintos productos que componen el índice general, afectando, como cabía esperar, en menor medida a los productos básicos. Así, mientras que las ventas de alimentos a precios constantes (corregidas de efectos estacionales y de calendario) aumentaron un $0,2 \%$ en el conjunto del año, las del resto de productos (que incluyen equipo personal, equipamiento del hogar y otros bienes) descendieron un 10,9\%.

El perfil de la serie general a lo largo de 2020 revela una abrupta interrupción de los incrementos interanuales que se venían registrando en enero y febrero, para pasar a descensos interanuales superiores al $31 \%$ en abril, coincidiendo con el confinamiento general, cuando las restricciones a la movilidad fueron más drásticas, y a descensos más moderados en la segunda parte del año. El mes de diciembre cerró con un descenso interanual del $1,5 \%$.

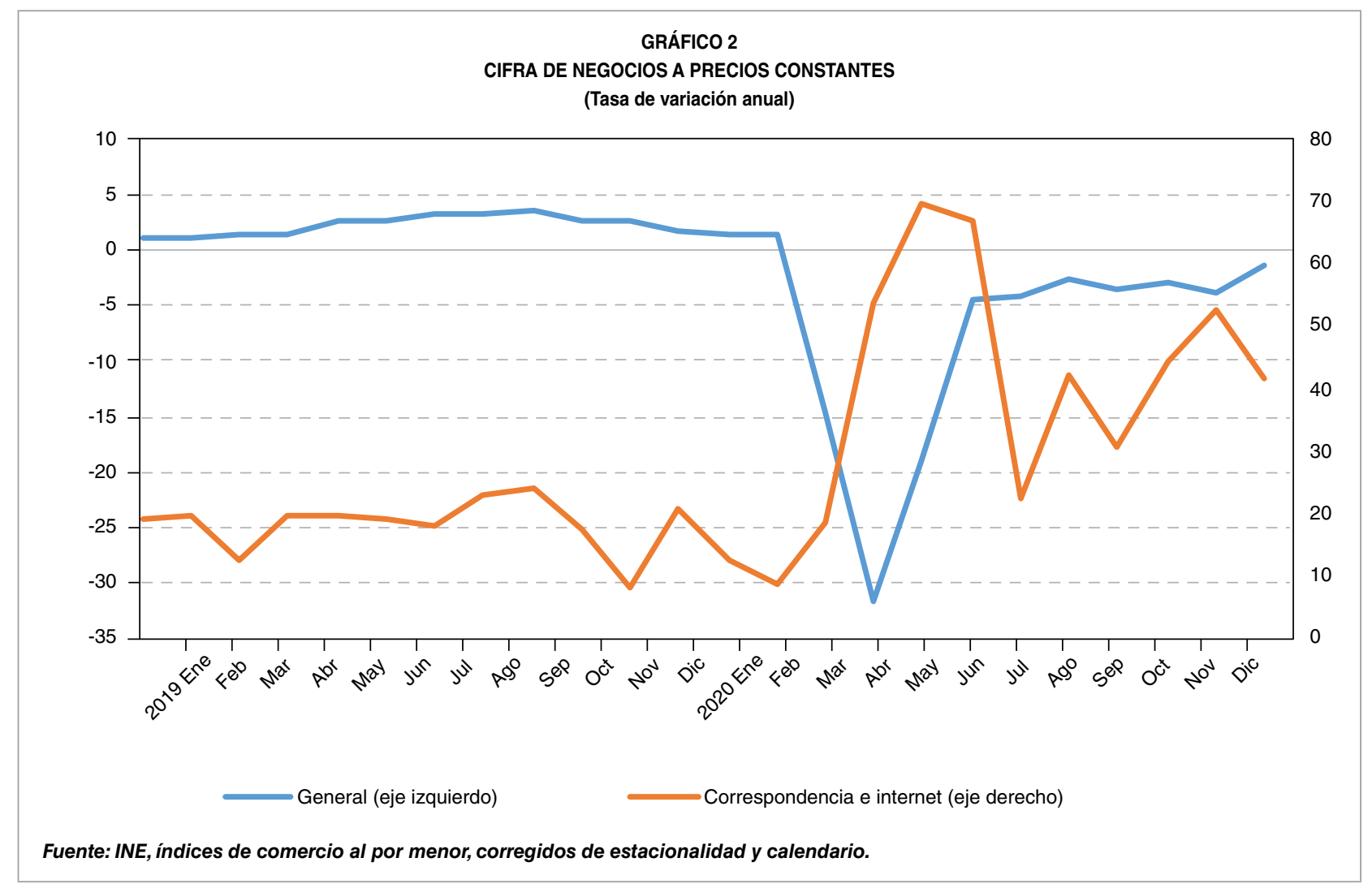


La evolución de las ventas de comercio al por menor de las empresas cuya actividad principal es la venta por internet o por correspondencia (clase 4791 de la CNAE) sugiere que la pandemia ha sido un factor acelerador de la tendencia de digitalización. En 2019, las ventas de estas empresas venían ya registrando crecimientos muy superiores a los del conjunto del sector, con tasas de variación interanual en el entorno del $20 \%$. En 2020 su crecimiento se ha acelerado, alcanzando tasas interanuales cercanas al $70 \%$ en los meses de mayo y junio y manteniéndose después, en promedio, en tasas próximas al 40\% (Gráfico 2). Las ventas de este subsector han presentado, por tanto, un perfil anual prácticamente opuesto al del conjunto del comercio al por menor y, además, parece que el efecto está teniendo una cierta permanencia. Esta evolución podría obedecer a un cambio del comportamiento de los consumidores, que, tras haber recurrido a las compras online en los meses de confinamiento, habrían incorporado esta práctica a sus hábitos de compra. En todo caso, este indicador solo aporta información sobre las ventas de las empresas cuya actividad principal se realiza online o por correspondencia. Será preciso atender a la evolución de otros indicadores de venta online, de espectro más amplio, para confirmar esta hipótesis.

El impacto de la pandemia en la actividad del comercio al por menor ha sido muy desigual entre las distintas regiones españolas. Esta heterogeneidad obedece a una diversidad de factores, entre los que cabe citar la propia evolución de la epidemia por territorios, las diferencias, entre las distintas comunidades autónomas, en la adopción de medidas de contención sanitaria y, sobre todo, el distinto grado de dependencia del turismo. En efecto, las restricciones nacionales $y$, sobre todo, internacionales a la movilidad han afectado especialmente al comercio minorista de los territorios más dependientes del turismo, como Canarias, Baleares, Cataluña o Andalucía. Similarmente, el comercio minorista de Ceuta y Melilla se ha visto especialmente afectado, dada su estrecha relación comercial con Marruecos, por el cierre de fronteras entre ese país y ambas ciudades autónomas, que se ha mantenido, ante la situación sanitaria, de forma prácticamente ininterrumpido desde el 13 de marzo de 2020.

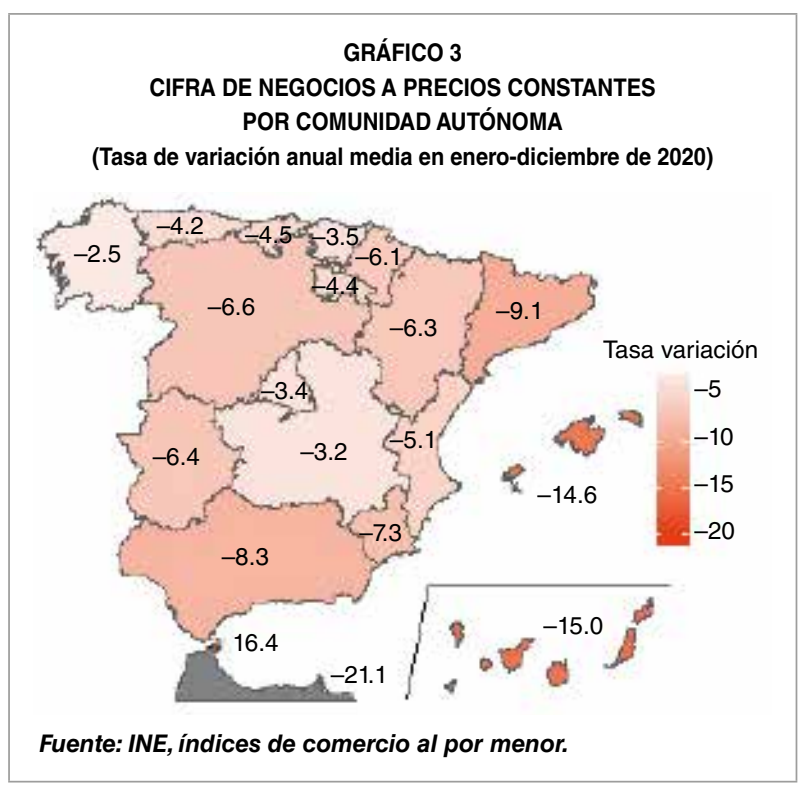

El descenso de la actividad también se ha reflejado en reducciones de la ocupación en el sector. Aunque el impacto en la evolución del empleo ha sido generalizado, el comportamiento según el modo de distribución ha registrado diferencias importantes.

Globalmente, con datos de la Encuesta de Población Activa (EPA), en el cuarto trimestre de 2020, el comercio al por menor registró 1.885.600 ocupados, 75.700 menos que en el mismo trimestre del año anterior (lo que supuso un descenso del 3,9\% interanual). La evolución del índice de comercio al por $\triangleright$ 
menor referido a ocupación muestra, igualmente, que, frente a un moderado crecimiento en 2019 , el año 2020 se ha caracterizado por descensos interanuales de la ocupación, que se iniciaron en marzo, al eclosionar la pandemia en España, y ha continuado en los siguientes meses, sin presentar indicios de recuperación.

Por modos de distribución, hay diferencias destacadas (Gráfico 4). Así, mientras que las grandes cadenas comerciales continuaron creando empleo durante la primera mitad del año y, en la segunda parte, registraron descensos relativamente moderados, las pequeñas cadenas son el modo de distribución que más ha sufrido en términos de empleo, registrando desde marzo fuertes caídas interanuales, que solo comenzaron a moderarse, ligeramente, en el mes de julio, para volver a intensificarse a final de año. Estos datos suponen profundizar en la tendencia ya observada en 2019, año en el que las grandes cadenas lideraron la creación de empleo en el sector, mientras que las pequeñas, junto con las grandes superficies, tuvieron un comportamiento menos favorable.

La evolución de la actividad y la ocupación en el comercio minorista, descrita en los párrafos anteriores, se explica, en buena medida, por la evolución de la COVID-19 en España y por la menor movilidad a causa de las medidas restrictivas que ha sido preciso imponer para su contención, así como por los cambios en los hábitos de los ciudadanos ante la elevada incidencia de esta enfermedad. Estas restricciones, junto con otros elementos, como la evolución de la actividad económica y del empleo generales, el aumento de ahorro precautorio y el descenso del turismo, han incidido de forma especialmente significativa en el gasto en consumo y, por lo tanto, en la actividad del sector.

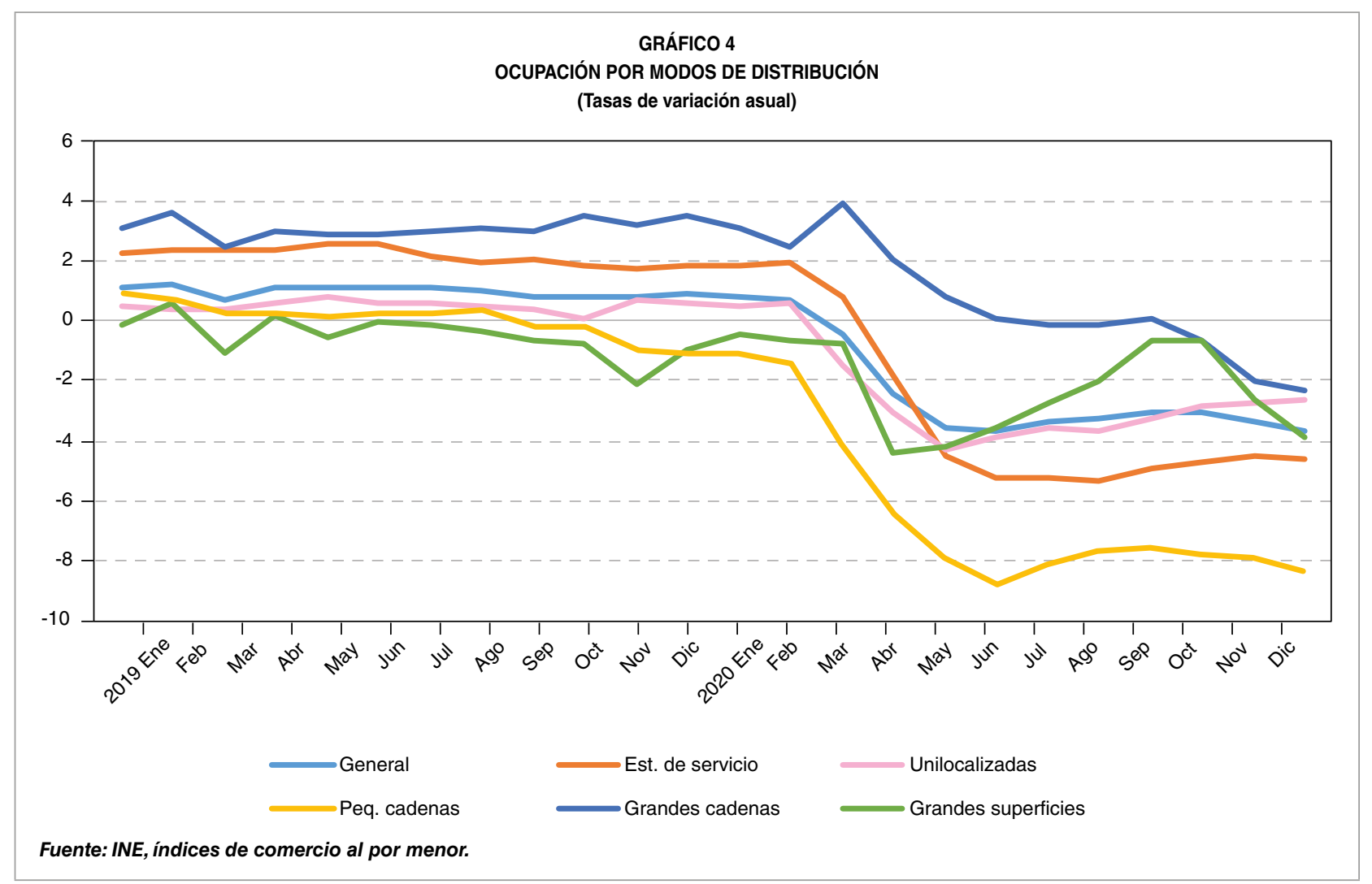


El gasto presencial mediante tarjetas de pago ha reflejado, desde la declaración del estado de alarma, las restricciones a la movilidad y a la actividad (González, 2020). Sobre esta base, y a partir de los datos de alta frecuencia de gasto presencial con tarjetas procedentes de BBVA y CaixaBank, la Secretaría de Estado de Comercio elabora un indicador sintético que aproxima la evolución del gasto en comercio al por menor (Subdirección General de Estudios y Evaluación de Instrumentos de Política Comercial, 2021). En el Gráfico 5 se muestra la evolución de dicho indicador, junto con la de casos notificados de COVID-19 y de indicadores de movilidad de Google referidos a comercio minorista y ocio, y alimentación y farmacia.

Las sucesivas oleadas de la pandemia se reflejan en el mencionado gráfico mediante los datos de casos notificados. Estos datos son los primeros conocidos por las autoridades sanitarias $y$, junto con otra información, como los datos de hospitalizaciones o ingresos en $\mathrm{UCI}$, que reflejan la evolución de la pandemia con más retraso, sirven de base para la adopción de medidas de contención. Al analizarlos, sin embargo, es preciso tener presente que se ven afectados por la capacidad de detección (asociada a la disponibilidad de test adecuados y de otros recursos sanitarios) que se ha incrementado sensiblemente desde el inicio de la pandemia, lo que explica en buena medida las grandes diferencias entre los datos comunicados en la primera oleada (que alcanza su máximo en la segunda mitad de marzo) frente a la segunda (con máximos a finales de octubre) y la tercera (en la que estamos inmersos en el momento de redactar este artículo).

Las sucesivas oleadas han venido acompañadas de medidas de contención que se han reflejado en restricciones a la movilidad. Estas restricciones, lógicamente, han tenido mayorimpacto en la movilidad asociada al comercio $D$

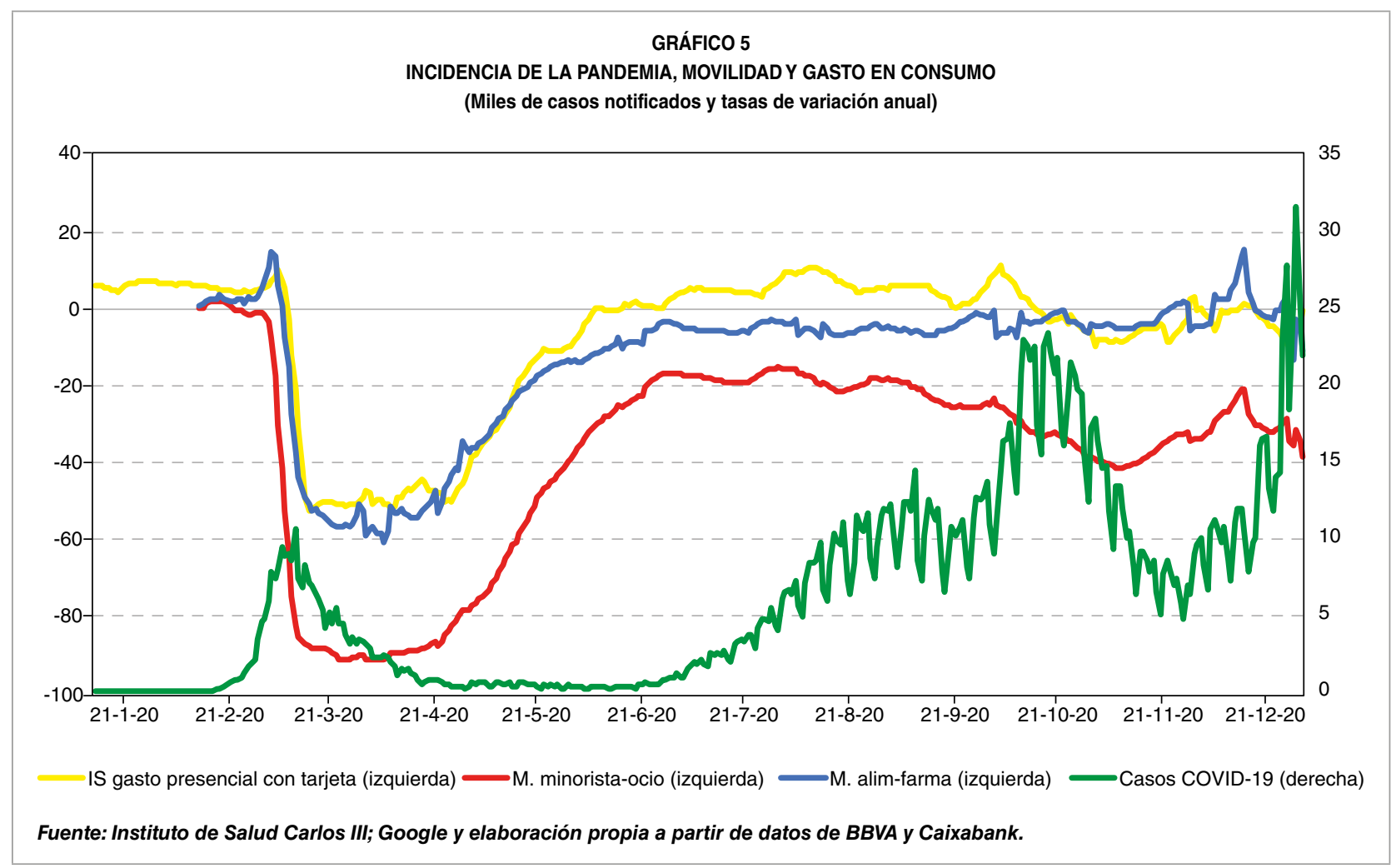




\section{ÍNDICES DE COMERCIO AL POR MENOR}

La encuesta de Índices de Comercio al por Menor, del Instituto Nacional de Estadística, tiene por objeto el conocimiento de las características fundamentales de las empresas dedicadas al ejercicio del comercio minorista en España y la medición, a corto plazo, de la evolución de la actividad del sector.

La población objeto de la encuesta está constituida por las empresas cuya actividad principal se inscribe en la división 47 de la sección G de la Clasificación Nacional de Actividades Económicas (CNAE-2009): comercio al por menor, excepto de vehículos de motor y motocicletas. Esta división comprende la reventa (venta sin transformación) de bienes nuevos y usados principalmente al público en general para su consumo o utilización personal o doméstica, en tiendas, grandes almacenes, puestos, empresas de venta por correo, vendedores a domicilio, vendedores ambulantes, economatos, etc.

Con relación a su ámbito temporal, los índices se elaboran con periodicidad mensual, y se refieren a un periodo base calculado como la media aritmética de los doce meses del año 2015. Se formulan como índices de Laspeyres encadenados que adoptan como periodo de referencia de las ponderaciones de cada año el mes de diciembre del año anterior al considerado.

Su ámbito geográfico es el de todas las unidades estadísticas ubicadas en el territorio del Estado español.

La encuesta ofrece información relativa a múltiples variables: económicas (cifra de negocios en términos nominales y a precios constantes), de empleo (personal ocupado, remunerado y no remunerado) y específicas del comercio (locales destinados a la venta, gran superficie, gran cadena, pequeña cadena, empresas unilocalizadas y estaciones de servicio, y comercio especializado y no especializado).

La encuesta permite un seguimiento de estas variables por tipo de producto, distinguiendo entre alimentación y resto, y, dentro de esta última categoría, entre equipo personal, equipamiento del hogar y otros bienes. También permite un seguimiento del comercio al por menor por comunidad y ciudad autónoma.

Fuente: INE (2020).

minorista general y al ocio (que incluye otras actividades, como restauración o espectáculos, también fuertemente afectadas por las restricciones) que a la asociada a actividades esenciales, como la farmacia y la alimentación, que habrían amortiguado, en cierta medida, el impacto económico de la pandemia en el conjunto del sector del comercio al por menor.
Las restricciones a la movilidad y el impacto en el consumo fueron especialmente significativos en la primera oleada. En la segunda y en la tercera se observan también descensos de la movilidad (fundamentalmente, de la asociada a actividades de comercio minorista y de ocio) que, si bien no han tenido un impacto tan significativo sobre el gasto presencial mediante tarjetas, han truncado la incipiente $D$ 
recuperación que se observaba desde los meses de verano.

En definitiva, el análisis de los indicadores disponibles revela que la COVID-19 y las medidas que se han adoptado para su contención han tenido un impacto elevado en la actividad del comercio al por menor que se ha reflejado tanto en las ventas como en la ocupación. Tras la primera oleada, el sector parecía haber iniciado una cierta recuperación, sin embargo, los repuntes de la pandemia han impedido que se consolidara.

\section{Las medidas paliativas de la crisis}

El análisis realizado es consistente con las expectativas existentes al inicio de la crisis sanitaria, de acuerdo con las cuales el sector de comercio minorista se configuraba como uno de los sectores económicos más susceptibles de resultar afectados por el impacto de la COVID-19, tanto por el impacto directo de las diversas medidas adoptadas para contención de la pandemia como por el efecto que las expectativas de las familias sobre la evolución de la economía y el empleo pueden tener sobre el consumo privado (Dirección General de Política Económica, 2020). En consecuencia, es también uno de los sectores que en mayor medida ha hecho uso de los instrumentos de apoyo diseñados para paliar el impacto económico de la crisis sanitaria.

La estrategia de política económica para mitigar los efectos de la crisis contempla dos fases conceptualmente diferenciadas, aunque relacionadas entre sí (Dirección General de Política Económica, 2020). En una primera fase, se ha tratado de garantizar la suficiencia de recursos para el sector sanitario y de proteger a los distintos agentes económicos (hogares y empresas), amortiguando así el impacto económico y social de la crisis. En esta fase, el comercio, junto con otros sectores especialmente afectados, como la hostelería y el turismo, ha recibido atención específica mediante medidas como las aprobadas en el Plan de Refuerzo a la Hostelería, el Comercio y el Turismo (instrumentado, principalmente, a través del RDL 35/2020).

En una segunda fase, cuando el shock sanitario pueda darse por concluido, el objetivo será la pronta recuperación de la actividad económica, persiguiendo que sea sólida, inclusiva y resiliente, y que dé respuesta, al mismo tiempo, a los retos que deberán afrontarse en los próximos años, en particular, la transformación tecnológica y digital y la transición ecológica (Plan de Recuperación, Transformación y Resiliencia de 2020).

En este epígrafe se analiza el uso por el sector de comercio minorista de algunas de las principales medidas adoptadas durante la primera fase. En primer lugar, se analiza el uso por el sector de las principales medidas de apoyo al sostenimiento del tejido productivo diseñadas para el conjunto de actividades económicas. En segundo lugar, se describen las principales medidas adoptadas, específicamente para el comercio y otros sectores especialmente afectados por la crisis, en diciembre de 2020. El reducido tiempo transcurrido desde su aprobación no permite, por el momento, una valoración de su utilización.

Las medidas de apoyo diseñadas para el conjunto de actividades económicas son susceptibles, en algunos casos, de ser aprovechadas tanto por los trabajadores autónomos como por otro tipo de empresas que desarrollan su actividad en el ámbito del comercio minorista. A grandes rasgos, pueden destacarse las siguientes líneas de actuación $\triangleright$ 
adoptadas a través de diversos instrumentos normativos ${ }^{1}$ :

- En el ámbito del apoyo financiero, además de líneas de financiación sectoriales específicas, o de la Línea COVID-19 de CERSA (Compañía Española de Reafianzamiento, SA), que potencia la cobertura por las sociedades de garantía recíproca de operaciones de financiación para pymes afectadas, destaca la línea de avales por un importe máximo de 100.000 millones de euros para cubrir, por cuenta del Estado, la financiación otorgada por entidades financieras. Los aplazamientos de deudas tributarias y las suspensiones de plazos en este ámbito persiguen también un alivio financiero para empresas, pymes y autónomos.

- En el ámbito laboral y de la Seguridad Social, se han adoptado medidas diversas, que incluyen la ampliación de bonificaciones a la Seguridad Social, moratorias y aplazamientos de pagos, o el establecimiento de una prestación extraordinaria por cese de actividad para autónomos. La medida más ampliamente utilizada ha sido el recurso a los Expedientes de Regulación Temporal del Empleo (ERTE).

- En otros ámbitos se incluyen una amplia variedad de medidas, como las que han afectado a los procedimientos de contratación pública, la interrupción de plazos para el deber de solicitar un concurso de

Dada la proliferación y complejidad de la normativa desarrollada para afrontar la pandemia y sus efectos, el Boletín Oficial del Estado ha publicado, como parte de su colección de Códigos Electrónicos, una serie de códigos que, bajo el título común COVID-19, agrupan las normas publicadas en los siguientes ámbitos: medidas tributarias, trabajadores autónomos, colectivos vulnerables, arrendamiento de vivienda y locales comerciales, derecho europeo y estatal, y derecho autonómico. Los códigos se mantienen permanentemente actualizados y pueden consultarse en:

https://www.boe.es/biblioteca_juridica/index.php?tipo $=C \&$ modo $=2$ acreedores, las orientadas a reducir costes energéticos, la ampliación de plazos para interponer recursos o reclamaciones, o las medidas adoptadas en materia de arrendamiento, que afectan también a arrendamientos de locales comerciales.

Entre los instrumentos que mayor alcance han tenido figuran la línea de avales vehiculada a través del Instituto de Crédito Oficial (ICO) y el recurso a los ERTE. En el Cuadro 4 y el Gráfico 6 se presentan los principales datos de utilización de estos instrumentos por parte del sector de comercio al por menor o, cuando no es posible presentar los datos con el nivel adecuado de desagregación, por el conjunto de actividades más relacionadas con este ámbito.

El objetivo de la línea de avales es cubrir, por cuenta del Estado, el riesgo asociado a la financiación que concedan las entidades financieras a las empresas y autónomos para atender sus necesidades de liquidez. Se aprobó en marzo de 2020 (RDL 8/2020), y su importe global se fijó en 100.000 millones de euros.

Las actividades relacionadas con el comercio minorista han sido usuarias intensivas de la línea de avales. Este uso es un reflejo de la magnitud que han tenido los efectos económicos de la pandemia en el sector, que determina una fuerte demanda de liquidez, y de las características de su tejido productivo, con fuerte presencia de pymes y autónomos, que determina a su vez dificultades de acceso a la financiación, paliadas por la existencia de los avales públicos.

Aunque no es posible establecer una correspondencia precisa entre comercio minorista, tal y como se define en la división 47 de la CNAE, y la clasificación sectorial que emplea el ICO para el seguimiento de la gestión de la línea $\triangleright$ 
(ICO, 2021), puede constatarse que las actividades que, en mayor o menor grado, guardan relación con este sector han absorbido una elevada proporción de los recursos movilizados. Así, más de 250.000 empresas de este ámbito, incluyendo también empresas no comerciales relacionadas con turismo y ocio, distribución comercial al por mayor y algunas actividades industriales relacionadas con textil y moda, se han beneficiado de la línea de avales. Esta cifra, que representa un $42 \%$ del total de empresas beneficiarias hasta finales de 2020 , se corresponde con un porcentaje ligeramente inferior de operaciones (38\%) y algo más reducido de avales solicitados y financiación concedida (en ambos casos, en torno al $32 \%$ ). Se trata, por lo tanto, de operaciones de importe inferior al promedio de la línea, lo que refleja la preponderancia de los autónomos y pymes en el sector, con capacidad de absorción de fondos más limitada que la de las empresas de mayor tamaño. En conjunto, se han solicitado, hasta finales de 2020, unos 27.700 millones de euros en avales que han permitido movilizar cerca de 37.000 millones de euros para la financiación de necesidades de liquidez de estas actividades relacionadas con el comercio interior.

El sector de comercio minorista ha sido también un usuario intensivo de los ERTE. En promedio, entre abril y diciembre de 2020 el porcentaje de los trabajadores procedentes del sector de comercio minorista (división 47 de la CNAE) acogidos a ERTE ha sido del 10,8\%, superior a la participación del sector en la ocupación (en torno al 9,8\%). Este dato es especialmente relevante si se tiene en cuenta la elevada participación de trabajadores autónomos en el sector, que por definición no pueden acogerse a ERTE, y de microempresas, para las que la carga burocrática de tramitación de un ERTE puede resultar una barrera difícil de superar.

El objetivo de este instrumento, cuyas condiciones de uso se han flexibilizado desde el inicio de la pandemia, es doble. Por un lado, protege a los trabajadores afectados, dándoles acceso a prestaciones por desempleo, manteniendo su vínculo con la empresa, y permitiendo que, en caso de que el ERTE $\square$

CUADRO 3

SITUACIÓN DE LA LÍNEA DE AVALES COVID-19 A 31 DE DICIEMBRE DE 2020

(Número de operaciones y empresas y millones de euros en avales y financiación)

\begin{tabular}{|c|c|c|c|c|}
\hline Línea de Avales COVID-19 & Operaciones & Empresas & Aval solicitado & Financiación concedida \\
\hline Actividades relacionadas con comercio interior .... & 359.642 & 250.807 & 27.740 & 36.689 \\
\hline Turismo, ocio y cultura ... & 173.395 & 125.262 & 12.821 & 17.177 \\
\hline Bienes de consumo y retail & 79.385 & 57.233 & 4.727 & 6.064 \\
\hline Distribución comercial de alimentos y bebidas .................. & 62.341 & 39.628 & 6.602 & 8.778 \\
\hline Industria textil y moda y retail. & 44.521 & 28.684 & 3.591 & 4.671 \\
\hline Total de la línea & 944.588 & 591.500 & 87.086 & 114.648 \\
\hline Porcentajes sobre el total de la línea & Operaciones & Empresas & Aval solicitado & Financiación concedida \\
\hline Actividades relacionadas con comercio interior & $38,1 \%$ & $42,4 \%$ & $31,9 \%$ & $32,0 \%$ \\
\hline Turismo, ocio y cultura & $18,4 \%$ & $21,2 \%$ & $14,7 \%$ & $15,0 \%$ \\
\hline Bienes de consumo y retail .... & $8,4 \%$ & $9,7 \%$ & $5,4 \%$ & $5,3 \%$ \\
\hline Distribución comercial de alimentos y bebidas .................. & $6,6 \%$ & $6,7 \%$ & $7,6 \%$ & $7,7 \%$ \\
\hline Industria textil y moda y retail & $4,7 \%$ & $4,8 \%$ & $4,1 \%$ & $4,1 \%$ \\
\hline Total de la línea & $100,0 \%$ & $100,0 \%$ & $100,0 \%$ & $100,0 \%$ \\
\hline
\end{tabular}


derive en una cesación definitiva de su relación contractual, mantengan sus derechos de acceso a prestaciones. Por otro lado, facilita a las empresas la suspensión temporal de la relación laboral ante una situación anómala de baja actividad que se prevé transitoria. De esta forma, se evitan costes asociados, bien al mantenimiento de los trabajadores en un momento de actividad reducida, bien a su despido, ante la incertidumbre sobre la duración de la situación. En definitiva, se trata tanto de proteger al trabajador como de preservar el tejido empresarial y los puestos de trabajo, facilitando así la posterior recuperación de la economía y el empleo.

Como puede observarse en el Gráfico 6, la evolución de los trabajadores acogidos a ERTE ha reflejado la trayectoria de la pandemia en España y de las medidas de restricción de la movilidad y la actividad adoptadas para su contención. En la primera oleada, este reflejo ha sido particularmente intenso en el comercio minorista, muy afectado en los momentos de mayor intensidad por las restricciones a la movilidad. Sin embargo, tras la desescalada e incluso durante la segunda y tercera oleadas, la participación de los trabajadores procedentes del comercio minorista sobre el total de los acogidos a ERTE ha descendido.

Así, durante la primera oleada (abril y mayo) el número de trabajadores acogidos a ERTE alcanza sus máximos, aproximándose a los 3,4 millones a finales de abril y principios de mayo. El máximo para el sector de comercio al por menor se alcanza también en estas fechas, con cifras próximas a los 450.000 trabajadores. El porcentaje de trabajadores procedentes del comercio al por menor dentro del total de los acogidos a ERTE se sitúa en el $13,8 \%$ y $12,6 \%$ en abril y mayo, respectivamente.

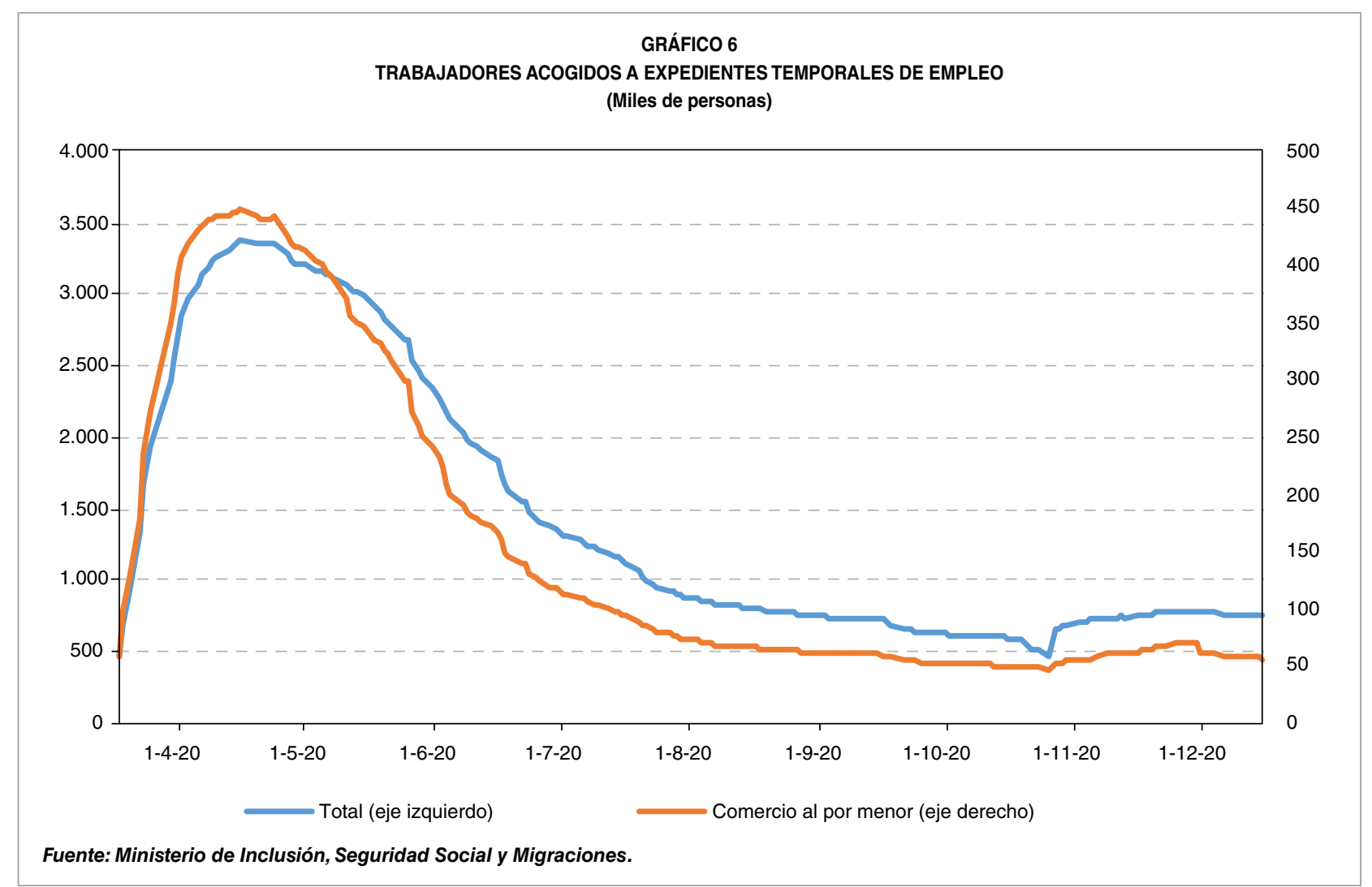


En la fase de desescalada, y hasta primeros de noviembre, se produce un descenso del número de trabajadores acogidos a ERTE, que llega a situarse en el entorno de los 500.000 para el total y de los 50.000 para el comercio al por menor. El porcentaje de los procedentes del comercio al por menor sobre el total desciende hasta el $8,3 \%$ de noviembre.

La segunda y la tercera oleadas han dado lugar a nuevos aumentos de estas cifras, que, a finales de 2020, superaban las 755.000 personas para el total general y las 56.000 para el comercio al por menor. Estos niveles son, en ambos casos, notablemente inferiores a los de la primera oleada, en la que, en el periodo de confinamiento generalizado, la restricción de la actividad fue muy intensa. El porcentaje que representan los trabajadores procedentes del comercio al por menor sobre el total de los acogidos a ERTE continuó descendiendo hasta el 8,2\% en diciembre.

En la segunda y la tercera oleadas, por tanto, el crecimiento del número de trabajadores acogidos a ERTE procedentes del comercio minorista ha sido mucho más suave que en la primera y menos acusado que en el conjunto de la actividad económica. Este comportamiento es consistente con el carácter menos restrictivo de las medidas de contención adoptadas y con una mejor preparación de las empresas para aplicar medidas de seguridad en los locales comerciales o utilizar otros canales de venta (por ejemplo, la venta electrónica o telefónica, con entrega a domicilio).

Como se ha señalado, el elevado impacto de la crisis sanitaria en el comercio minorista y su sensibilidad ante las medidas adoptadas para contener la pandemia ha determinado, al igual que en los ámbitos de la hostelería o el turismo, la necesidad de adoptar medidas de apoyo y refuerzo específicas. Entre ellas, las acciones aprobadas en el Plan de Refuerzo a la Hostelería, el Comercio y el Turismo, adoptado por el Consejo de Ministros el 22 de diciembre de 2020. Alguna de las medidas que se incluyen en este plan habían sido impulsadas en meses previos ante su urgencia. El objetivo común de estas medidas es apoyar a empresas y autónomos de estos sectores, facilitando que, en la situación de baja actividad debida a la pandemia, puedan atender sus costes fijos (costes laborales, alquileres, impuestos, etc.) que, si no se recibieran los apoyos, pondrían en riesgo la viabilidad de las empresas y la futura recuperación de la actividad y el empleo. Las medidas adoptadas pueden agruparse en cinco ejes, que se describen a continuación, señalando las principales medidas de apoyo al comercio minorista (no se incluyen, por lo tanto, las específicamente destinadas a hostelería y turismo).

- Arrendamientos de locales comerciales. Se incentiva el acuerdo entre los arrendadores y arrendatarios para reducir o aplazar pagos. Si el arrendador es un gran tenedor de locales comerciales (posee diez o más), a falta de acuerdo, el arrendatario puede optar por una reducción del 50\% del alquiler, durante el estado de alarma, sus prórrogas y hasta cuatro meses más, o por una moratoria durante el mismo plazo, con un periodo de dos años a partir de su finalización para el pago aplazado de las rentas. Se estima que el número de locales comerciales potencialmente beneficiados asciende a 157.000 , lo que supone cerca del $29 \%$ del total de locales dedicados a comercio minorista. Para los pequeños arrendadores se establece un incentivo fiscal que impulsa la reducción voluntaria de los alquileres de locales comerciales durante el primer trimestre de 2021. 
- Apoyo a la liquidez y solvencia. Se amplían tanto el periodo de carencia (en un máximo de un año) como los plazos de amortización (en un máximo de tres años) de los préstamos garantizados por la línea de avales o por las sociedades de garantía recíproca que cuentan con apoyo de CERSA. Estas medidas se adoptaron por el Consejo de Ministros el 24 de noviembre de 2020.

- Ámbito tributario. Entre las diversas medidas que se adoptan destaca el nuevo aplazamiento de deudas tributarias (por un máximo de seis meses, con tres de carencia de intereses), con el objetivo de evitar tensiones de tesorería. Se estima que podrían beneficiarse de la medida 68.000 contribuyentes del sector de comercio al por menor. Además, se incrementa la reducción en la tributación por módulos del impuesto sobre la renta de las personas físicas. Esta reducción se eleva con carácter general del $5 \%$ hasta el $20 \%$ y para los sectores más afectados, como el comercio, hasta el $35 \%$. Se aplica también para el régimen simplificado del IVA. La reducción se aplica al conjunto de 2020 y al primer trimestre de 2021.

- Ámbito laboral y de la Seguridad Social. Se establecen también aplazamientos de los pagos de cotizaciones a la Seguridad Social para las cuotas devengadas entre diciembre de 2020 y febrero de 2021 (para empresas) o de enero a marzo de 2021 (para autónomos). El sector de comercio (igual que los de turismo y hostelería) podrá aplicar una bonificación del 50\% en las cuotas de los contratos de fijos discontinuos de abril a octubre de 2021.

- Medidas regulatorias. En este ámbito se adoptan medidas de orientación general que pueden beneficiar en algún caso al sector de comercio minorista. Así, se flexibilizan requisitos para mantener los incentivos regionales de proyectos de inversión en marcha o en fase de supervisión de los compromisos de mantenimiento del empleo y de la inversión que los titulares de los proyectos asumen cuando acceden a las subvenciones. Además, se amplía la moratoria para presentar concurso de acreedores y se establece un régimen transitorio para las zonas de gran afluencia turística, evitando que la reducción del número de turistas por efecto de la pandemia resulte en un régimen más restrictivo para la actividad comercial de estas áreas.

\section{Conclusiones: hacia la recuperación y transformación}

La pandemia COVID-19 ha dejado una profunda huella en el sector del comercio minorista. Las medidas restrictivas de la movilidad, necesarias para frenar la expansión del virus, han tenido un efecto especialmente intenso en la actividad de comercio al por menor que se ha visto también afectado por el descenso del turismo. Este impacto tiene, además, un efecto negativo en términos de igualdad de género, dado el papel del sector como generador de empleo femenino y fuente de oportunidades de emprendimiento para las mujeres. La estructura empresarial del sector, atomizado y con fuerte predominio del trabajo autónomo y la microempresa, lo hace especialmente vulnerable a este impacto, por lo que ha requerido un apoyo público también intenso.

Las medidas de apoyo al comercio minorista analizadas en este artículo se han adoptado con el fin de suavizar en lo posible este $\triangleright$ 
impacto económico, social y territorial y, especialmente, de preservar el tejido empresarial. El objetivo es que, de esta forma, cuando se inicie la recuperación, esta sea rápida y venga acompañada también de una pronta mejora del empleo en el sector.

Ahora bien, es preciso tener presente que, al recuperar la senda de crecimiento, el comercio minorista deberá afrontar el reto de modernización y transformación digital en el que estaba ya inmerso antes de la pandemia. La propia crisis sanitaria puede haber acelerado la tendencia de cambio y modificado hábitos de consumo, haciendo más urgente y necesaria la transformación del comercio minorista. En este desafío, el sector requerirá el apoyo de las políticas públicas.

El Ministerio de Industria, Comercio y Turismo, como responsable del diseño de las políticas orientadas al sector, es consciente, desde hace años, de esta necesidad. Muestra de ello fue la creación del Observatorio del Comercio 4.0, mediante Acuerdo del Consejo de Ministros de 11 de octubre de 2018, como grupo de trabajo, consultivo, asesor y de colaboración entre la Administración General del Estado y las organizaciones y asociaciones representativas del comercio y de la economía digital (publicado por Resolución de 25 de octubre de 2018, de la Secretaría de Estado de Comercio). El objetivo de este observatorio es servir de apoyo a los principales agentes del sector para el análisis y comprensión de la transformación digital, así como en la definición de las estrategias, iniciativas y políticas, públicas o privadas, más adecuadas para afrontarla.

En la reunión virtual del Observatorio del Comercio 4.0, celebrada el 16 de diciembre de 2020, se presentó un plan de impulso del sector comercial, enmarcado en el Plan de Recuperación, Transformación y Resiliencia, que movilizará 317 millones de euros procedentes de los fondos europeos, durante el periodo 2021-2023. Los ejes en torno a los cuales se articulará el plan incluyen: la digitalización del sector; su sostenibilidad social y medio ambiental; el impulso a la gobernanza, competitividad y competencia leal; la capacitación, formación e información; y la recuperación de la confianza y sensibilidad del sector.

En definitiva, el diseño de las nuevas políticas de apoyo orientadas al comercio minorista debe atender no solo a su recuperación, sino también a la superación de los grandes retos que afronta el sector. Entre ellos destaca el de su transformación digital y el de alinear esta transformación con los objetivos de mantener su papel clave como vector de cohesión social y territorial y de contribuir al crecimiento inclusivo y sostenible.

\section{Bibliografía}

Dirección General de Política Económica (2020). Política económica ante la emergencia del coronavirus. Boletín Económico de ICE (3122). https://doi.org/10.32796/bice.2020.3122.6997

González Mínguez, J. M., Urtasun Amann, A., y Pérez García de Mirasierra, M. (2020). Evolución del consumo en España durante la vigencia del estado de alarma: un análisis a partir del gasto con tarjetas de pago. Boletín económico del Banco de España. Artículos Analíticos (3). https://repositorio.bde.es/bitstream/123456789/ 13241/1/be2003-at22.pdf

Instituto de Crédito Oficial (2021). Informe Línea Avales COVID a 31 de diciembre de 2020. https://www.ico.es/documents/19/2429886/Infor$\mathrm{me}+\mathrm{L} \% \mathrm{C} 3 \%$ ADnea+Avales $+311220 /$ a506f93b-9eb7-447e-9159-164f3b677893

Instituto Nacional de Estadística (2020). Índices de Comercio al por Menor. Base 2015. Manual metodológico. https://www.ine.es/daco/daco43/notaccm15.pdf 
Las gasolineras automáticas crecen un $17 \%$, hasta superar los 1.000 puntos, y alcanzan una cuota del $10 \%$ (18 de junio de 2020). Europa Press. https://www.europapress.es/economia/energia-00341/noticia-gasolineras-automaticas-crecen-17-superar-1000-puntos-alcanzan-cuota-10-20200618142427.html

Németh Kecskeméti, G. (2020). El potencial efecto competitivo de las estaciones de servicio automáticas en España. Boletín Económico de ICE (3126). https://doi.org/10.32796/bice.2020.3126. 7071

Observatorio Nacional de las Telecomunicaciones y de la Sociedad de la Información (2020). El comercio electrónico B2C en España. https://www. ontsi.red.es/sites/ontsi/files/2020-11/B2C2019_ Ed2020_0.pdf

Presidencia del Gobierno (2020). Plan de Recuperación, Transformación y Resiliencia. https:// www.lamoncloa.gob.es/presidente/actividades/ Documents/2020/07102020_PlanRecuperacion. pdf

Real Decreto Ley 8/2020, de 17 de marzo, de medidas urgentes extraordinarias para hacer frente al impacto económico y social del COVID-19. Boletín Oficial del Estado, n. ${ }^{\circ}$ 73, de 18 de marzo de
2020, pp. 25853 a 25898. https://www.boe.es/eli/ es/rdl/2020/03/17/8

Real Decreto Ley 35/2020, de 22 de diciembre, de medidas urgentes de apoyo al sector turístico, la hostelería y el comercio en materia tributaria. Boletín Oficial del Estado, n.․ 334, de 23 de diciembre de 2020, pp. 118678 a 118707. https:// www.boe.es/eli/es/rdl/2020/12/22/35/con

Resolución de 25 de octubre de 2018, de la Secretaría de Estado de Comercio, por la que se publica el Acuerdo del Consejo de Ministros de 11 de octubre de 2018, por el que se crea el Observatorio del Comercio 4.0. Boletín Oficial del Estado n. -279 , de 19 de noviembre de 2018, pp 112317 a 112322. https://www.boe.es/eli/es/res/2018/10/ $25 /(3)$

Subdirección General de Estudios y Evaluación de Instrumentos de Política Comercial (2021). Cuaderno de Indicadores de Comercio Interior. https://comercio.gob.es/Comerciolnterior/indicadores/Paginas/indicadores-comercio-interior. aspx

Subdirección General de Comercio Internacional de Servicios y Comercio Digital (2019). El retail en España, cada vez menos interior, cada vez más digital. Boletín Económico de ICE (3114). http:// www.revistasice.com/index.php/BICE/article/ view/6893 
